\title{
A patient and physician survey of fibromyalgia across Latin America and Europe
}

\author{
Patricia Clark ${ }^{1}$ Eduardo S Paiva ${ }^{2}$, Anna Ginovker ${ }^{3}$ and Patricia Arline Salomón ${ }^{4^{*}}$
}

\begin{abstract}
Background: Patients and physicians from three Latin American (LA) and six European countries were surveyed in order to describe differences in journey to diagnosis, impact, and management of fibromyalgia (FM).

Methods: 900 patients (300 LA; 600 Europe) and 1824 physicians (604 LA; 1220 Europe) were surveyed between October-December 2010 (LA) and February-April 2008 (Europe). Patients and physicians (GP or specialists) completed separate questionnaires, on symptoms, impact, and FM management. Interviews were conducted in local languages. Appropriate rating scales were used throughout. Data were analyzed using cross-tabulations and descriptive statistics. Significance was determined at $P<0.05$ (indicated by *).
\end{abstract}

Results: In LA versus Europe, patients reported having FM symptoms for longer (100.8 vs. 83.7* months), and taking longer to be diagnosed (42.3 vs. 31.1* months). FM was characterized by multiple symptoms (11.2 vs. 6.9), but more LA patients reported 14 common symptoms*, and rated pain higher on 11-point scale (8.0 vs. $\left.7.2^{*}\right)$. LA patients were taking fewer medications (3.3 vs. 4.0). Patients from both regions found common symptoms very/extremely disruptive to their quality of life, but symptoms impacted daily living and ability to work more significantly in LA. Physicians (GPs or specialists) from LA more often considered problems sleeping*, difficulty concentrating*, anxiety*, depression*, numbness/tingling*, and leg cramps* very/extremely disruptive vs. European physicians. Despite headache, heightened sensitivity to touch, difficulty concentrating, and joint pain being experienced by $\geq 50 \%$ of patients from both regions, $<15 \%$ of PCPs or specialists considered these typical FM symptoms. Patients also considered 12/14 symptoms more disruptive than PCPs or specialists in the same region. However, a higher proportion of PCPs or specialists considered FM to have a strong/very strong impact on aspects of daily living vs. patients within the same region.

Conclusions: Patient- and physician-rated disease perception and impact was often higher in LA than in Europe. Patient and physician perspective concerning FM impact and disruption were often misaligned within the same region. Our observations may be representative of cultural differences in stoicism, expression, beliefs, and attitudes to pain perception and management. Better understanding of these complexities could help targeted educational/ training programs incorporating cultural differences, to improve chronic care.

Keywords: Survey data, Impact of fibromyalgia, Regional comparison, Burden of disease

\footnotetext{
* Correspondence: patricia.salomon@pfizer.com

${ }^{4}$ Pfizer Inc, Paseo de los Tamarindos \#40 Col. Bosques de las Lomas, DF, 05120, Mexico, Mexico

Full list of author information is available at the end of the article
} 


\section{Background}

Fibromyalgia (FM) is a multi-factorial disease involving physiological as well as psychological factors, and characterized by widespread pain and muscle tenderness accompanied by other comorbid symptoms [1,2]. Prevalence estimates vary, with up to $5 \%$ of women reported to have FM from survey data in the US and across Europe [2-4], but some lower estimates have been presented from survey data of rheumatic diseases for other regions, such as certain Latin American countries [5-9]. Although the reasons for differences in prevalence are ultimately unknown, differences among healthcare practices, historical recognition of FM symptoms, and dissemination of guidelines on diagnosis in different regions of the world could be factors. In addition, referral bias, whereby hospital-based data produce higher estimates of disease prevalence than survey samples from the general population, also contributes to variation in the literature.

Guidelines for diagnosis and/or management of FM have been published by recognized bodies, such as the American College of Rheumatology (ACR) [1,10], and by bodies outside of the US [11-13]. Despite these protocols, FM is under-diagnosed and under-treated [14]. Physicians may diagnose patients by ruling out other conditions that share some symptoms with FM, for example chronic fatigue syndrome, rheumatoid arthritis, and multiple sclerosis [15]. Local treatment practices and diagnosis guidelines especially outside of the US and the ACR may not be widely disseminated to physicians who encounter patients with FM symptoms in their everyday practice. As a result, diagnosis and treatment can be delayed and efforts to improve recognition and diagnosis are needed [14]. For some patients, satisfaction with health status increases with a diagnosis [16], and therefore earlier diagnosis and treatment may improve treatment response and reduce the negative impact that FM symptoms have on aspects of daily living [17-20]. FM patients in some countries report frequent healthcare use and work days lost compared with both non-FM subjects and patients with other rheumatic conditions [17,21-23]. As a result of high healthcare use, studies from the US and Europe have found that FM places a significant economic burden on patients and healthcare systems [24-26]. Hidden costs of disability and comorbidities associated with FM likely increase the true burden of FM even higher.

Despite the well-reported burden FM has on activities of daily living, few data on the social and personal impact of FM on patients from Latin American countries have been published, and no data, to our knowledge, have been published examining patient and physician data from Latin America in comparison with other countries that use different healthcare practices. This international survey of FM patients and physicians from three Latin American countries and six European countries sought to examine differences in the perception and management of FM, and social and occupational aspects of FM between countries with different cultures and economies: Latin America and Europe.

\section{Methods}

Data were collected between February-April 2008 in Europe, and October-December 2010 in Latin America. Surveyed physicians including primary care practitioners (PCPs) and specialists were identified using proprietary physician databases developed and maintained by research agencies in each of the countries, lists of professional physician associations, phone directories, and other commercially available sample sources. "Physicians who treated FM patients" or "FM-treating physicians" were defined for this survey as physicians currently seeing or who had seen at least one FM patient over the past 2 years. Patients had to have been diagnosed with FM by a physician and they were identified by physicians who treated them for FM. The recruiting physicians either participated in the survey or were sampled specifically to recruit FM patients, but did not complete the physician survey. Except for patients from Brazil, patients and physicians were compensated for completing the survey. Ethical approval for this study was not required according to regulations within the countries, at the time the surveys were carried out.

\section{Questionnaires}

Patients and physicians completed different surveys. The sponsor had no role during the conduct of the survey, and the sponsor's name did not appear on any of the survey materials, nor was it mentioned to patients or physicians during the survey. Therefore, the influence of the sponsor on responses by physicians or patients would be negligible. The English questionnaire was translated into each language by an independent professional translation agency, and all translations were then reviewed by a separate, independent translation agency. The trained interviewer in healthcare research who administered the questionnaire also reviewed the translations before collecting data. Patient interviews (faceto-face or via telephone) averaged 25 minutes and were conducted in local languages. Rating scales were used throughout the survey.

Questions covered symptoms (a given list of 14 common symptoms), management, and impact of FM. Physicians were also asked questions regarding their clinical background. The scales used were: Disruption Scale (Extremely disruptive, Very disruptive, Fairly disruptive, Not very disruptive, Not at all disruptive; top two box ratings $=$ Extremely/Very disruptive); Agreement Scale 
(Agree strongly, Agree somewhat, Neither agree nor disagree, Disagree somewhat, Disagree strongly; top two box ratings $=$ Agree strongly/Agree somewhat); Impact Scale (Very strong impact, Strong impact, Moderate impact, Slight impact, No impact; top two box ratings = Very strong/Strong impact); Satisfaction Scale (Extremely satisfied, Very satisfied, Satisfied, Somewhat satisfied, Not at all satisfied; top two box ratings = Extremely satisfied/Very satisfied). Pain was rated on an 11-point numerical rating scale from 0 ("No pain") to 10 ("Worst possible pain"). Copies of the surveys can be found in the Additional file 1 and Additional file 2. All scales had the equivalent option of decline to answer or "Not known/Unsure".

\section{Statistical testing}

Data were processed and quality assured. Data were analyzed using cross-tabulations and descriptive statistics; no multivariate analysis was done. Significance was determined at $P<0.05$ using $t$ test of column proportions.

\section{Results}

\section{Patient's perspective of FM}

The study sample included 900 patients with a confirmed diagnosis of FM according to ACR 1990 criteria [10]; 300 patients from Latin America (100 each from Mexico, Venezuela, Brazil), and 600 from Europe (100 each from UK, France, Germany, Italy, Spain, the Netherlands). The majority of patients were female (93\% LA; 85\% Europe) and aged 45-59 years (Table 1). Patients from Latin America reported having FM symptoms for a significantly longer time (100.8 vs. 83.7 months), and taking significantly longer to be diagnosed (42.3 vs. 31.1 months), and seeing more physicians to receive a diagnosis $(5.4$ vs. 4.0 physicians) compared with European patients, respectively $(P<0.05$ for all). In a typical month, fewer patients from Latin America vs. Europe visited their physicians $\geq 2$ / month $(23 \%$ vs. $44 \% ; P<0.05)$.

FM was characterized by multiple symptoms in both regions (LA: 11.2; Europe: 6.9), but a significantly higher proportion of patients from Latin America vs. Europe, respectively, reported common symptoms, including widespread pain ( $92 \%$ vs. $62 \%$ ), sleep problems ( $84 \%$ vs. $49 \%$ ), and fatigue ( $88 \%$ vs. $46 \%$ ) (Table 1). Patients from Latin America also rated their pain higher on an 11-point scale vs. European patients (8.0 vs. 7.2). Patients from both regions reported common FM symptoms as disruptive (very/extremely), most commonly chronic/widespread pain (LA vs. Europe: $86 \%$ vs. $78 \%$ ), sleeping problems ( $80 \%$ vs. $76 \%$ ), and fatigue ( $80 \%$ vs. $75 \%$ ) (Table 2). Patients from Latin America more often reported that FM impacted their ability to work and/or earn income than European patients (Figure 1). Although fewer patients from Latin America vs. Europe had been unemployed during the past
Table 1 Patient characteristics and FM symptoms reported by patients from Latin America and Europe

\begin{tabular}{|c|c|c|}
\hline $\begin{array}{l}\text { Patient characteristics or } \\
\text { symptoms }(\%)\end{array}$ & $\begin{array}{l}\text { Latin America } \\
(\mathrm{N}=300)\end{array}$ & $\begin{array}{l}\text { Europe } \\
(N=600)\end{array}$ \\
\hline Gender, Female & $93 \%$ & $85 \%$ \\
\hline \multicolumn{3}{|l|}{ Age, years } \\
\hline $18-35$ & $19 \%$ & $22 \%$ \\
\hline $36-44$ & $21 \%$ & $25 \%$ \\
\hline $45-59$ & $41 \%$ & $33 \%$ \\
\hline $60-74$ & $16 \%$ & $17 \%$ \\
\hline $75+$ & $1 \%$ & $4 \%$ \\
\hline Average number of symptoms & 11.2 & 6.9 \\
\hline \multicolumn{3}{|l|}{ Common symptoms experienced $^{a}$} \\
\hline Chronic/widespread pain & $92 \% *$ & $62 \%$ \\
\hline Problems sleeping & $84 \% *$ & $49 \%$ \\
\hline Fatigue & $88 \% *$ & $46 \%$ \\
\hline Headaches & $84 \% *$ & $61 \%$ \\
\hline Facial pain & $52 \% *$ & $42 \%$ \\
\hline Heightened sensitivity to touch & $73 \% *$ & $50 \%$ \\
\hline Difficulty concentrating & $78 \% *$ & $52 \%$ \\
\hline Numbness \&/or tingling sensations & $82 \% *$ & $42 \%$ \\
\hline Feelings of anxiety & $79 \% *$ & $28 \%$ \\
\hline Feelings of depression & $80 \% *$ & $41 \%$ \\
\hline Joint pain & $89 \% *$ & $59 \%$ \\
\hline Stiffness & $77 \% *$ & $49 \%$ \\
\hline Leg cramps & $75 \% *$ & $47 \%$ \\
\hline Low back pain & $83 \% *$ & $55 \%$ \\
\hline
\end{tabular}

a 14 common symptoms were given.

${ }^{*} P<0.05$.

12-months (LA: 33\%, Europe: 42\%); significantly more patients from Latin America reported missing $\geq 40$ days of work due to FM (LA 24\%; Europe: 3\%). Patients from Latin America also more often reported that FM had a strong or very strong impact on aspects of daily living than those from Europe, including on physical mobility, motivation/drive, and their overall quality of life (Figure 2A).

On average, patients from Latin America were taking fewer medications (mean 3.3) than their European counterparts (mean 4.0). When asked what treatments they were currently using for FM, a similar proportion of patients from both regions were taking analgesics prescribed by physicians (LA: 66\% vs. Europe: 70\%), but more patients from Europe were taking over-the-counter (OTC) pain relievers (LA: 27\% vs. Europe: $44 \%: P<$ 0.05) (Figure 3). Patients from Latin America were less likely than European patients to be using certain nonpharmacological therapies for their FM, including relaxation techniques, biofeedback, and lifestyle changes (Figure 3; $P<0.05$ for all). Despite reporting use of 
Table 2 Proportion of patients, PCPs, and specialists who report each symptom as "Very" or "Extremely" disruptive

\begin{tabular}{|c|c|c|c|c|c|c|}
\hline \multirow[b]{2}{*}{ Common symptoms } & \multicolumn{2}{|c|}{ Patients (\%) } & \multicolumn{2}{|c|}{ PCPs $(\%)^{a}$} & \multicolumn{2}{|c|}{ Specialists (\%) ${ }^{a}$} \\
\hline & $\begin{array}{c}\text { Latin America } \\
(\mathrm{N}=300)\end{array}$ & $\begin{array}{c}\text { Europe } \\
(\mathrm{N}=600)\end{array}$ & $\begin{array}{c}\text { Latin America } \\
(\mathrm{N}=253)\end{array}$ & $\begin{array}{c}\text { Europe } \\
(\mathrm{N}=503)\end{array}$ & $\begin{array}{l}\text { Latin America } \\
(\mathrm{N}=254)\end{array}$ & $\begin{array}{c}\text { Europe } \\
(\mathrm{N}=495)\end{array}$ \\
\hline Chronic/Widespread pain & $86 \% * \neq$ & $78 \%$ & $79 \%$ & $78 \%$ & $80 \%$ & $79 \%$ \\
\hline Problems sleeping & $80 \% \ddagger \S$ & $76 \% \neq \S$ & $70 \% *$ & $50 \%$ & $69 \% *$ & $55 \%$ \\
\hline Fatigue & $80 \% \ddagger \S$ & $75 \% \neq \S$ & $69 \%$ & $67 \%$ & $66 \%$ & $69 \%$ \\
\hline Headaches & $69 \% \neq \S$ & $77 \% * \neq \S$ & $52 \% *$ & $37 \%$ & $45 \%$ & $38 \%$ \\
\hline Facial pain & $59 \% \neq \S$ & $69 \%^{*} \neq \S$ & $40 \% *+$ & $24 \%$ & $30 \%$ & $27 \%$ \\
\hline Heightened sensitivity to touch & $61 \% \neq \S$ & $72 \%^{*} \neq \S$ & $50 \% *$ & $42 \%$ & $48 \%$ & $49 \%+$ \\
\hline Difficulty concentrating & $73 \% \neq \S$ & $73 \% \neq \S$ & $51 \% *$ & $36 \%$ & $50 \% *$ & $39 \%$ \\
\hline Numbness \&/or tingling sensations & $59 \% \neq \S$ & $62 \% \neq \S$ & $48 \% *+$ & $25 \%$ & $37 \% *$ & $24 \%$ \\
\hline Feelings of anxiety & $75 \% * \ddagger \S$ & $64 \% \ddagger \S$ & $66 \% *$ & $44 \%$ & $64 \% *$ & $47 \%$ \\
\hline Feelings of depression & $73 \%$ & $77 \% \neq \S$ & $74 \% *$ & $58 \%$ & $73 \% *$ & $62 \%$ \\
\hline Joint pain & $80 \% \neq \S$ & $76 \% \neq \S$ & $68 \% *+$ & $55 \%$ & $54 \%$ & $53 \%$ \\
\hline Stiffness & $74 \% \ddagger \S$ & $74 \% \neq \S$ & $59 \% *+$ & $39 \%$ & $44 \%$ & $39 \%$ \\
\hline Leg cramps & $67 \% * \neq \S$ & $60 \% \ddagger \S$ & $42 \% *$ & $24 \%$ & $35 \% *$ & $27 \%$ \\
\hline Low back pain & $81 \% * \neq \S$ & $75 \% \neq \S$ & $59 \% *+$ & $44 \%$ & $50 \%$ & $45 \%$ \\
\hline
\end{tabular}

${ }^{a}$ Base case: Physicians currently seeing or have seen patients with $\mathrm{FM} ;{ }^{*}=P<0.05$ Europe vs. Latin America within group $(\mathrm{Patient}, \mathrm{PCP}, \mathrm{Specialist)}+\mathrm{P}<0.05 \mathrm{PCP}$ vs. specialist (within region); $\neq P<0.05$ patient vs. $\mathrm{PCP}$ (within region); $\S P<0.05$ patient vs. specialist (within region).

various pharmacological and non-pharmacological therapies for FM, only $30 \%$ of Latin American patients and $20 \%$ of European patients were satisfied (extremely/ very) with the ability of their treatments to relieve FM.

\section{Physicians' perspective of FM}

The study sample included 1824 physicians (604 LA; 1220 Europe). PCPs were general/family practitioners (LA 68\%; Europe 100\%) and the remainder were internists (LA 32\%). Specialists were neurologists (LA: 29\%; Europe: 25\%), psychiatrists (LA: 26\%; Europe: 28\%), rheumatologists (LA: 27\%; Europe: 26\%), pain specialists/anaesthetists (LA: 16\%; Europe 13\%) and internists (LA: 1\%; Europe 2\%). The majority of physicians had been in practice for 11-30 years across regions, and were treating $\leq 20 \mathrm{FM}$ patients, regardless of speciality (Table 3). PCPs saw more patients per month in each region than specialists, but, as would be expected, specialists in each region saw more FM patients than PCP. The majority of physicians were aware of 1990 ACR criteria, although the proportion of PCPs and specialists aware of and working in practices that employed these criteria was greater in Latin America than in Europe. Of 14 common symptoms, physicians similarly considered chronic/widespread pain a typical symptom in Latin America and in Europe, for PCPs (79\% vs. 78\%) and specialists $(80 \%$ vs. $79 \%)$, respectively. Both sets of physicians from Latin America more often considered problems sleeping, difficulty concentrating, anxiety, depression, numbness/tingling, and leg cramps as disruptive (very/ extremely) vs. European physicians (Table 2). More than
$82 \%$ of PCPs and specialists from both regions indicated that FM has a very strong or strong impact on patients overall quality of life (Figure 2B and C). More PCPs from Latin America than from Europe, however, considered FM to have a very strong or strong impact on the individual aspects of daily living (Figure 2B). Generally, similar proportions of specialists from Latin America vs. Europe rated impact on aspects of daily living (Figure 2C). When compared within Europe, differences were recorded between PCPs and specialists responses on the impact that FM has aspects of daily living. Within Latin America the responses from PCPs and specialists were similar for all questions of daily living, except for the impact of FM on sex life (Figure 2B and C).

\section{Examining patient and physician perspectives}

Chronic/widespread pain was a common symptom experienced by the majority of patients, and was considered a typical FM symptom by the majority of physicians (PCPs, and specialists, respectively) from both regions. But, despite headache, heightened sensitivity to touch, difficulty concentrating, and joint pain being experienced by $\geq 50 \%$ of patients from both regions (Table 1 ), $<15 \%$ of PCPs or specialists considered these typical FM symptoms (Table 4).

Chronic/widespread pain was considered disruptive (very/extremely) by $\geq 78 \%$ of patients, PCPs and specialists from both regions. Opinions on the disruption caused by the majority of the other 14 listed FM symptoms were not aligned among patients and PCPs or specialists within the same region. For example, significantly more patients 


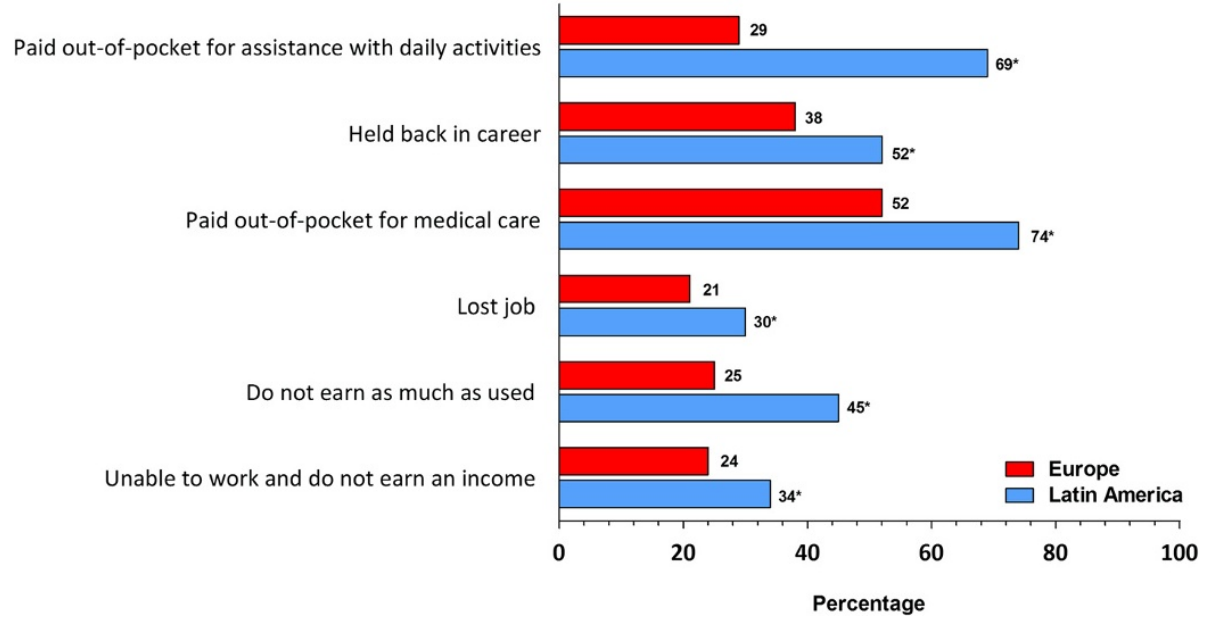

Figure 1 Patient-reported impact of FM on work status and income. ${ }^{*}=P<0.05$ comparison Europe vs. Latin America.

within Latin America and within Europe considered at least 12/14 symptoms more disruptive than PCPs or specialists within the same region (Table 2). The proportion of patients and physicians (PCPs or specialists) within the same region also differed on the impact caused by certain
FM symptoms on aspects of daily living but, interestingly, a higher proportion of PCPs from Latin America, or specialists from both regions, considered FM had a strong or very strong impact on aspects of daily living compared with patients within the same region (Figure 2).

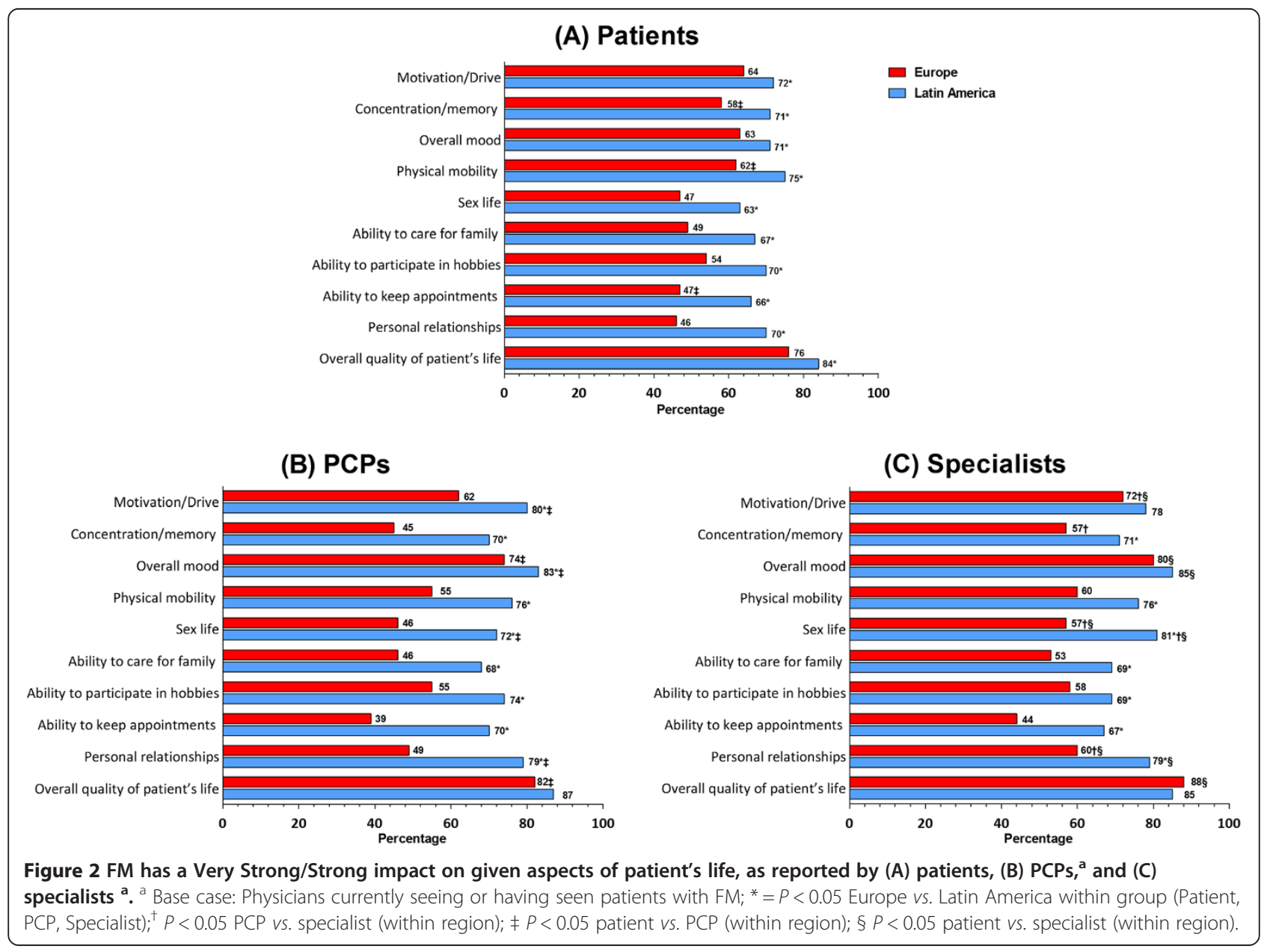




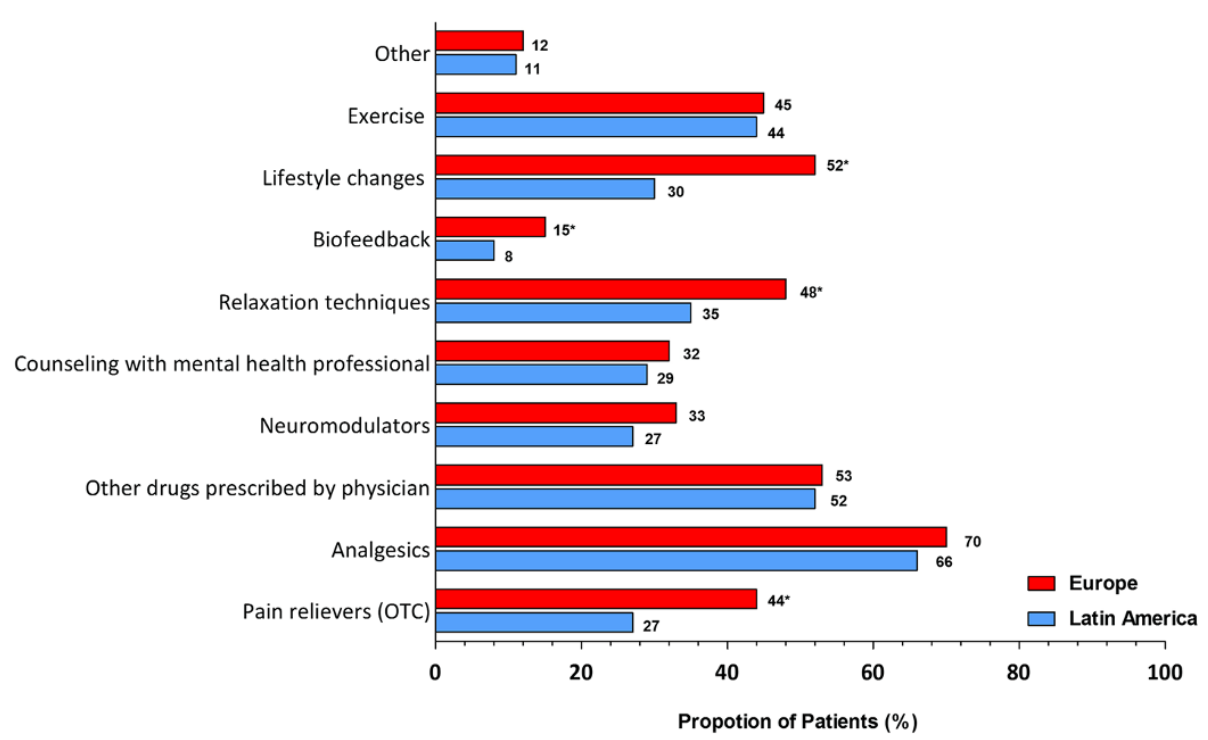

Figure 3 Pharmacological and non-pharmacological treatments that patients most frequently reported receiving to treat their FM. ${ }^{*}=P<0.05$ comparison Europe vs. Latin America. OTC, over the counter.

\section{Discussion}

A wealth of data exists on the burden of FM from individual countries, particularly from the United States. However data from within the Latin American region and healthcare setting is particularly sparse [27] and no study has described the Latin American perspective of FM alongside patients from a different region. This study describes how FM is perceived and managed across different healthcare settings using two regions with different cultures and economies: Latin America and Europe.

Table 3 Selected physician characteristics

\begin{tabular}{|c|c|c|c|c|}
\hline & \multicolumn{2}{|l|}{ Europe } & \multicolumn{2}{|c|}{ Latin America } \\
\hline & PCP & Specialist & PCP & Specialist \\
\hline & $N=609$ & $N=611$ & $N=306$ & $N=298$ \\
\hline Years in practice & $21.2^{*}$ & 19.7 & 16.5 & $18.4^{*}$ \\
\hline $1-10$ years & $103(16.9 \%)$ & $136(22.3 \%)$ & $123(40.2 \%)$ & $90(30.2 \%)$ \\
\hline $11-30$ years & $434(71.3 \%)$ & $384(62.8 \%)$ & $155(50.7 \%)$ & $161(54.0 \%$ \\
\hline$\geq 31$ years & $71+(11.7 \%)$ & $91(14.9 \%)$ & $28(9.2 \%)$ & $43+(14.4 \%)$ \\
\hline How many patients do you see per month & $473.1^{*}$ & 250.3 & $271.5^{*}$ & 191.8 \\
\hline$\leq 100$ & $60(9.9 \%)$ & $171(28.0 \%)$ & $77(25.2 \%)$ & $121(40.6 \%$ \\
\hline $101-300$ & $153(25.1 \%)$ & $275(45.0 \%)$ & $144(47.1 \%)$ & $112(37.6 \%$ \\
\hline$\geq 300$ & $392+(65.0 \%)$ & $163+(26.7 \%)$ & $81+(26.5 \%)$ & $60+(20.1 \%$ \\
\hline Have seen FM patients in past 2 years (including currently) & $n=503$ & $n=495$ & $n=253$ & $n=254$ \\
\hline How many FM patients have you seen in the past 2 years (including currently) ${ }^{a}$ & 20.0 & $61.0^{*}$ & 53.9 & $145.7^{*}$ \\
\hline $1-20$ & $401(79.7 \%)$ & $270(54.5 \%)$ & $169(66.8 \%)$ & $140(55.1 \%$ \\
\hline $21-100$ & $72(14.3 \%)$ & $150(30.3 \%)$ & $57(22.5 \%)$ & $71(28.0 \%)$ \\
\hline$\geq 101$ & $28+(5.6 \%)$ & $73+(14.7 \%)$ & $26+(10.3 \%)$ & $42+(16.5 \%$ \\
\hline \multicolumn{5}{|l|}{ Are you aware of ACR 1990 criteria } \\
\hline Yes & $249(40.9 \%)$ & $341(55.8 \%)$ & $221(72.2 \%)$ & $227(76.2 \%$ \\
\hline \multicolumn{5}{|l|}{ Does your practice use ACR 1990 criteria to diagnose FM patients } \\
\hline Yes & $151(24.8 \%)$ & $218(35.7 \%)$ & $165(53.9 \%)$ & $184(61.7 \%$ \\
\hline
\end{tabular}

* significant difference PCP vs. specialists within region.

${ }^{\text {a }}$ use base case $=$ currently seeing/have seen FM patients in past 2 years.

† remainder declined to answer. 
Table 4 Typical symptoms that physicians look for when diagnosing $\mathrm{FM}$

\begin{tabular}{|c|c|c|c|c|}
\hline \multirow{3}{*}{ Symptom ${ }^{+}$} & \multicolumn{2}{|c|}{ Europe } & \multicolumn{2}{|c|}{ Latin America } \\
\hline & PCP & Specialist & PCP & Specialist \\
\hline & $\mathrm{N}=503$ & $\mathrm{~N}=495$ & $N=253$ & $N=254$ \\
\hline $\begin{array}{l}\text { Chronic/widespread } \\
\text { pain }\end{array}$ & $55 \%$ & $54 \%$ & $68 \%$ & $74 \%$ \\
\hline Problems sleeping & $1 \%$ & $2 \%$ & $4 \%$ & $2 \%$ \\
\hline Fatigue & $7 \%$ & $9 \%$ & $5 \%$ & $4 \%$ \\
\hline Headaches & 0 & 0 & 0 & $1 \%$ \\
\hline Facial pain & 0 & $1 \%$ & $2 \%$ & 0 \\
\hline $\begin{array}{l}\text { Heightened sensitivity } \\
\text { to touch }\end{array}$ & $14 \%$ & $14 \%$ & $7 \%$ & $5 \%$ \\
\hline Difficulty concentrating & $1 \%$ & $1 \%$ & 0 & 0 \\
\hline $\begin{array}{l}\text { Numbness \&/or } \\
\text { tingling sensations }\end{array}$ & $3 \%$ & $2 \%$ & $2 \%$ & $1 \%$ \\
\hline Feelings of anxiety & 0 & $1 \%$ & 0 & 0 \\
\hline Feelings of depression & $4 \%$ & $4 \%$ & $2 \%$ & $1 \%$ \\
\hline Joint pain & $7 \%$ & $5 \%$ & $3 \%$ & $3 \%$ \\
\hline Stiffness & $3 \%$ & $2 \%$ & $2 \%$ & $2 \%$ \\
\hline Leg cramps & 0 & $1 \%$ & $1 \%$ & 0 \\
\hline Low back pain & $3 \%$ & $1 \%$ & $3 \%$ & $3 \%$ \\
\hline
\end{tabular}

${ }^{\dagger} 14$ common symptoms were given. Base case $=$ physicians currently seeing or having seen patients with FM.

Consistent with reports from other countries [17,22,28], FM is reported by patients and physicians to be a debilitating chronic/widespread pain condition, more common in women, and which is characterized by multiple symptoms that negatively impact daily living. The negative impact FM had on patients and the disruption symptoms caused to aspects of daily living are also broadly in line with survey data from other regions [17,19,20,22], and reinforce the high burden FM imparts on patients, regardless of region of origin. Given the range of the data surveyed, some divergence would be expected. Nevertheless, our study presents many new observations in these populations, in addition to highlighting important differences, particularly between the impact and disruption caused by FM from a physician and patient viewpoint, within the same region. Our data therefore highlight some misalignment in perceptions of FM that need to be addressed and warrant further investigation.

Our analysis extends individual reports from within Latin America or Europe [5,7,19,29] to describe differences between patient and physician experiences regarding how FM is diagnosed, perceived, and managed. For example, patients from Latin America reported having FM symptoms for significantly longer time, and taking significantly longer to be diagnosed, and seeing more physicians to receive a diagnosis compared with European patients. Studies from the United States indicate that patients often go 5 years before receiving a correct diagnosis of FM $[14,17,30]$, suggesting that despite the differences in journey to diagnosis patients from both regions herein were diagnosed relatively quickly (LA: 3.5 years; Europe: 2.5 years). The time difference seen between regions is representative of the work-up patients with FM face when a diagnosis is unclear, particularly historically when FM was less well defined and understood. For example, pain or other symptoms (eg., fatigue, sleep problems, depression, etc.) may have been treated by physicians as separate conditions, or even disregarded if they considered there to be a lack of medical evidence for the pain [31]. These and similar issues are inherent variables some patients face prior to receiving a confirmed diagnosis of FM [14]. Physicians, both PCPs and specialists, were generally aware of the ACR 1990 diagnosis guidelines; however they were more widely known and adopted in Latin America vs. European practices. This could be representative of European practices adopting European diagnosis or management practices that are more locally disseminated to PCPs and specialists [32]. Evidence suggests that receiving an accurate diagnosis is the first step to effective care and better outcomes for patients [14]. As such, differences in time to diagnosis in Latin America compared with Europe may have influenced disease characteristics. For example, a higher proportion of patients from Latin America reported common symptoms, and reported symptoms as being disruptive, than their European counterparts. Other factors influencing journey to diagnosis and FM experience between regions should also be considered, including patients being considered in different countries the responsibility of primary care vs. rheumatology or other specialist, which will in turn influence treatment practices as discussed in detail below.

Data were collected across different countries and healthcare systems that have different treatment practices and cultures and some of the differences observed in the FM experience may be reflective of these cultures and practices. For example, although some data have reported that clinical characteristics and patient perceptions are broadly similar across different regions $[17,33,34]$, important ethnic, racial, and sociocultural differences between populations in pain perception have been noted [35-38]. Given the ethnic diversity of populations surveyed, these factors may well have influenced responses provided, and account for some of the differences in disease experiences recorded, although the magnitude of these influences remains unknown. Zborowski and Zola published pioneering work investigating ethnic and cultural differences in the experience of pain, including attitudes and behavioral aspects that are generated after pain, the differences in impact of the disease, and what patients from different ethnic backgrounds do in 
order to find help or a cure for their pain [39,40]. Since this early work, numerous authors have published cultural differences regarding stoicism, expression, beliefs, and attitudes with regard to pain and pain perception. The ways in which patients and healthcare providers interact is also influenced by ethnicity, particularly when a painful condition is present [41], and in certain ethnic groups pain is reportedly undertreated [42]. For many questions in the present study, patients from Latin America rated responses significantly higher than in Europe, which may be representative of the more "expressive" nature of patients from Latin American and the more stoic European population [41]. How these differences in journey to diagnosis and expression of FM may ultimately impact FM prevalence, which is generally lower in Latin American countries compared with Europe $[3,5,9,29,43]$, would need further study. As such, the generalizability of the data to other countries or other FM patient populations cannot be assumed.

Patients from both regions reported using pharmacological and non-pharmacological approaches to manage their FM symptoms, and differences noted between regions provide some important insights into healthcare practices between Europe and Latin America. For example, aerobic exercise is helpful for some FM patients $[44,45]$ and it is somewhat surprising that less than $50 \%$ of patients reported exercise therapy in either region. This may suggest that when appropriate, physicians should encourage their patients to participate in some form of exercise. An interesting area of further study in these regions would be to categorize the type and/or schedule of exercise, e.g., giving subtypes of exercise (gentle, aerobic, walking, etc.) may change the proportion of patients responding. For example, a study comparing FM management in Germany vs. the US reported that "aerobic exercise" was used by $32 \%$ of the US consumers compared with 58\% German consumers; however gentle exercise was much higher (64\% vs 80\%) [46]. Other non-pharmacological treatment options, such as relaxation techniques, biofeedback, and lifestyle changes, were more commonly used in Europe. Given differences in economy for some Latin American vs. European countries, these treatments may not be easily accessible and/or reimbursed, and their utility and effectiveness is therefore harder to demonstrate in Latin America. From a pharmacological perspective, FM was managed by multiple medications, albeit fewer in Latin America than in Europe, which as noted above, may have influenced number and negative impact of FM symptoms in Latin America vs. Europe. Physician treatment practices have been shown to vary depending on ethnicity $[42,47]$. These inherent variables may have influenced prescribing practices in Latin America vs. Europe, especially considering fewer symptoms were experienced by patients in Europe.
OTC medications were used by up to $70 \%$ of patients in both regions, highlighting that patients may have been self-managing their symptoms, in addition to, or instead of their prescriptions. Although the specific OTC therapies used were not captured and will have varied among countries surveyed even within-region, over half of all patients reported out-of-pocket costs in both regions, albeit significantly more in patients from Latin America than Europe. Although not quantified by actual costs, the wider economic impact of FM was most notable in Latin America, with almost half of employed patients missing more than 40 days' work in the past year. Efforts should be made to help keep patients working, as long-time sick leave has been found detrimental to long-term recovery $[48,49]$. As a result, energy conservation, occupational therapy, evaluation, and work adaptation are important aspects of FM management $[49,50]$ and our survey suggests in particular that physicians in Latin America should be made aware and help keep patients in work.

We report that many different types of physicians are seeing FM patients in these two healthcare settings, probably due to the diverse range of symptoms that are involved in the condition. Our study highlights that in Europe more patients were seen in primary care by a general practitioner, compared with $32 \%$ seen by internists for primary care in Latin America. A range of specialists were seeing FM patients in both regions. The ability to conduct an efficient and accurate assessment and diagnosis will depend on the physician's background knowledge and training [51,52], and the differences in training experiences between countries may have influenced patients' journey to diagnosis, as discussed previously. For example, ACR diagnosis criteria are used in many countries outside of the United States, including most often in Latin American countries and across some parts of Europe. Regional knowledge of diagnosis guidelines will vary, however. Recent advances in the understanding of the pathophysiology of FM may not have extended to nonspecialists in primary care, and may also have differed between regions as indicated by the differences in knowledge and adoption of the ACR criteria, for example. In addition, updated ACR diagnosis criteria [1] and other guidelines outside of the US $[12,32]$ reflect that generalized pain does not adequately characterize FM and a broader assessment of pain, function, and psychosocial aspects may aid in FM diagnosis and, ultimately, management. These observations are supported by the symptoms experienced by patients from both regions in the present survey. Updated diagnosis guidelines were released by ACR in May 2010, just prior to the Latin American survey. As a result, these updated guidelines may have increased general awareness of FM. Indeed, although pain was the principal symptom in both regions, Latin American physicians more often considered a wider range of typical FM symptoms as 
"Very" or "Extremely" disruptive to their patients than their European counterparts, many of which are consistent with the updated ACR diagnosis guidelines. With growing acceptance and understanding of FM, there seems a need to include focused education for physicians regarding FM, starting ideally at medical school, to ensure all types of physician have a basic understanding of the condition when encountered. It is also paramount there is a better understanding, particularly in non-specialists, of mechanisms of chronic vs. acute pain in order to fully understand the physiology of FM and other chronic pain conditions. This is particularly important given the different approaches to management required for pain of different origins, in addition to effective management of comorbidities and the multi-factorial symptoms of FM [53]. Enhancing educational initiatives in both countries may help improve recognition, diagnosis, and patient satisfaction with management in the longer term [54-56]. We also suggest that further efforts to implement structured treatment paradigms, incorporating pharmacological and non-pharmacological approaches for FM, may likewise guide physicians and improve confidence in treatment choices most appropriate for FM.

It is well known that a range of physicians encounter patients with FM; however, few studies have commented on how patients and physicians perceive FM differently and furthermore how this is influenced by physician type. Overall, FM symptoms were reported by patients and physicians from both regions to be "Very" or "Extremely" disruptive, and having a negative impact on many aspects of daily living. More physicians (PCPs or specialists) from Latin America considered recognized somatic symptoms of FM (per $[1,10]$ ), such as problems sleeping, difficulty concentrating, depression, and numbness/tingling, as disruptive (Very/Extremely) than their European counterparts. Nevertheless, more Latin American patients rated FM symptoms as disruptive than their physicians. This suggests that although physicians in Latin America are more aware of the disruption caused by common FM symptoms than their European counterparts, their perceptions still fall short of patients from the same region. Despite these differences in opinions on disruption of FM symptoms, interestingly within Latin America, more physicians (PCPs or specialists) rated that FM had a "Very strong" or "Strong" impact on mood, sex life, and personal relationships than patients themselves, and furthermore matched patient's responses to all other aspects of daily living. Within Europe, more specialists likewise rated that FM had a very strong or strong impact on some aspects of daily living than European patients themselves, although responses from patients and PCPs in Europe were generally better aligned. Our observations suggest effort is needed to align patient's and physician's perception of FM within these regions. For example, quality of life questionnaires alongside educational initiatives could help draw attention to which FM symptoms are most disruptive and important to the patient. These types of questionnaires are not widely used in private practice, despite being translated into different languages [57-60]. Collectively, our observations indicate that there is still a long way to go concerning optimizing management of FM, irrespective of ethnic background and prescribing practices.

Our study should be interpreted in light of some limitations. The World Health Organization (WHO) recognized and classified fibromyalgia in 1993, yet in some countries doctors will have been unfamiliar with the condition, diagnosis criteria and/or treatment practices. In some countries included in our survey, no treatments are specifically approved for the treatment of FM and therefore management of patients both with prescription products and off label where no products are specifically available may have differed, particularly to countries where clear treatment protocols for FM are accepted and recommended. We sought to ensure that the diagnosis of FM was not entirely dependent on the patient's recollection, by only including patients who were diagnosed with FM by a physician according to standard criteria [10]. FM is a clinical diagnosis and previous research suggests physicians express difficulties in diagnosis and managing FM, and confidence varied depending on specialty [51]. Therefore whether or not physicians were board-certified, and also if physicians had experience of FM sufficient to make a differential diagnosis, are inherent variables for some of the questions posed in the survey. We therefore ensured that medical evidence was available for FM being present, to limit the possibility of physicians disregarding the pain condition due to lack of medical evidence [31]. An important limitation of all opinion research, to which this study is not an exception, is that respondents may not perfectly recall their experiences and feelings at the time of the survey, as respondents' feelings, attitudes, and perceptions are subject to change with time. The survey only provides a snapshot of the respondents' experiences and does not seek to address how these might have changed longitudinally. Therefore, the observations should be considered within the time-frame they were captured, which differed slightly between regions as noted above. Although the numbers of respondents per country were the same, data were collected from more European countries than Latin American countries, increasing the diversity of responders within the European dataset. Despite these limitations, our study provides new insights into the perceptions and management of FM patients in countries outside of the US, and identified some potential areas for targeted improvement. 


\section{Conclusions}

Differences between FM characteristics, treatment practices, and opinions were noted by physicians and patients from Latin America and Europe and interestingly patient- and physician-rated disease impact and perception was often higher in Latin America than in Europe. Improved understanding of these complexities involved in FM in different healthcare settings may help target educational/training programs towards improving aspects of chronic care. As patient's and physician's perspectives concerning FM impact and disruption were often misaligned within the same region, there is a clear need to focus on understanding and ultimately improving these conflicting views in order to optimize chronic care.

\section{Additional files}

Additional file 1: Physician questionnaire.

Additional file 2: Patient questionnaire a ${ }^{\text {a }}$ Published previously by: Choy E, Perrot S, Leon T, Kaplan J, Petersel D, Ginovker A, Kramer E: A patient survey of the impact of fibromyalgia and the journey to diagnosis. BMC Health Serv Res 2010, 10:102 [17].

\section{Competing interests}

PATRICIA CLARK, MD, PHD: In the past years, Dr. Clark has received consulting fees from Pfizer related to epidemiology work Dr. Clark is not involved in drug research or consultancy; Dr Clark does not hold stocks from any company and is not applying for any patents at the time of submission. EDUARDO S. PAIVA, MD: In the past five years, Dr Paiva has received fees for lectures in medications used in the treatment of fibromyalgia, from Pfizer Inc and Lilly, and has received fees from Pfizer Inc to present findings of this survey to journalists. Dr Paiva does not holds stocks from any company and is not applying for any patents at the time of submission.

ANNA GINOVKER, PHD: of Harris Interactive was a paid consultant to Pfizer Inc in connection with the conduct of this study.

PATRICIA ARLINE SALOMÓN, MD: is an employee of Pfizer Inc and hold stock options from Pfizer Inc.

\section{Authors' contributions}

AG was involved in the conception and design of the survey. All authors were involved in either the acquisition or analysis and interpretation of the data. All authors were involved in drafting the manuscript and revising it critically during development. All authors read and approved the final draft of the manuscript.

\section{Authors' information}

$P C$ is head of the Clinical Epidemiology Unit, and the Hospital Infantil Federico Gómez-faculty of Medicne UNAM; and an International Fellow at the American College of Rheumatology. ESP is an Assistant Professor at the Universidade Federal do Parana, Curitiba, Brazil; Chief, Fibromyalgia Clinic, Universidade Federal do Parana, Curitiba, Brazil; and an International Fellow at the American College of Rheumatology.

\section{Acknowledgments}

This study was sponsored by Pfizer Inc, who were involved in the design and conduct of the study. Medical writing support was provided by Karen Burrows, MPhil, of UBC Scientific Solutions and was funded by Pfizer Inc

\section{Sources of funding}

This study was sponsored by Pfizer Inc. The sponsor's name did not appear on the Survey materials.

\section{Author details}

${ }^{1}$ Clinical Epidemiology Unit HIM-Federico Gómez Faculty of Medicine UNAM, Colonia Doctores, DF, Mexico City, Mexico. ${ }^{2}$ Hospital de Clinicas, Universidade Federal do Parana, Hospital de Clinicas, Curitiba, Brazil. ${ }^{3}$ Harris Interactive, Inc., Rochester, New York, NY, USA. ${ }^{4}$ Pfizer Inc, Paseo de los Tamarindos \#40 Col. Bosques de las Lomas, DF, 05120, Mexico, Mexico.

Received: 10 October 2012 Accepted: 29 May 2013

Published: 14 June 2013

\section{References}

1. Wolfe F, Clauw DJ, Fitzcharles MA, Goldenberg DL, Katz RS, Mease P, Russell AS, Russell IJ, Winfield JB, Yunus MB: The American College of Rheumatology preliminary diagnostic criteria for fibromyalgia and measurement of symptom severity. Arthritis Care Res 2010, 62(5):600-610.

2. Wolfe F, Ross K, Anderson J, Russell IJ, Hebert L: The prevalence and characteristics of fibromyalgia in the general population. Arthritis Rheum 1995, 38(1):19-28.

3. Branco JC, Bannwarth B, Failde I, Abello Carbonell J, Blotman F, Spaeth M, Saraiva F, Nacci F, Thomas E, Caubere JP, et al: Prevalence of fibromyalgia: a survey in five European countries. Semin Arthritis Rheum 2010, 39(6):448-453.

4. Bennett RM, Jones J, Turk DC, Russell IJ, Matallana L: An internet survey of 2,596 people with fibromyalgia. BMC Musculoskelet Disord 2007, 8:27.

5. Peláez-Ballestas I, Sanin LH, Moreno-Montoya J, Alvarez-Nemegyei J, BurgosVargas R, Garza-Elizondo M, Rodríguez-Amado J, Goycochea-Robles MV, Madariaga M, Zamudio J, et al: Epidemiology of the rheumatic diseases in Mexico. A study of 5 regions based on the COPCORD methodology. J Rheumatol Supp/ 2011, 86:3-8.

6. Rodríguez-Amado J, Pelàez-Ballestas I, Sanin LH, Esquivel-Valerio JA, BurgosVargas R, Perez-Barbosa L, Riega-Torres J, Garza-Elizondo MA: Epidemiology of rheumatic diseases. A community-based study in urban and rural populations in the state of Nuevo Leon, Mexico. J Rheumatol 2011, 38:9-14.

7. Alvarez-Nemegyei J, Peláez-Ballestas I, Rodríguez-Amado J, Sanin LH, GarciaGarcia C, Garza-Elizondo MA, Loyola-Sanchez A, Burgos-Vargas R, Goycochea-Robles MV: Prevalence of rheumatic regional pain syndromes in adults from Mexico: a community survey using COPCORD for screening and syndrome-specific diagnostic criteria. J Rheumatol Suppl 2011, 86:15-20.

8. Senna ER, De Barros AL, Silva EO, Costa IF, Pereira LV, Ciconelli RM, Ferraz $M B$ : Prevalence of rheumatic diseases in Brazil: a study using the COPCORD approach. J Rheumatol 2004, 31(3):594-597.

9. Assumpção A, Cavalcante AB, Capela CE, Sauer JF, Chalot SD, Pereira CAB, Marques AP: Prevalence of fibromyalgia in a low socioeconomic status population. BMC Musculoskelet Disord 2009, 10:64.

10. Wolfe F, Smythe HA, Yunus MB, Bennett RM, Bombardier C, Goldenberg DL, Tugwell P, Campbell SM, Abeles M, Clark P, et al: The American College of Rheumatology 1990 Criteria for the Classification of Fibromyalgia. Report of the Multicenter Criteria Committee. Arthritis Rheum 1990, 33(2):160-172.

11. Carville SF, Arendt-Nielsen S, Bliddal H, Blotman F, Branco JC, Buskila D, Da Silva JA, Danneskiold-Samsoe B, Dincer F, Henriksson C, et al: EULAR evidence-based recommendations for the management of fibromyalgia syndrome. Ann Rheum Dis 2008, 67(4):536-541.

12. Fitzcharles MA, Ste-Marie PA, Goldenberg DL, Pereira JX, Abbey S, Choinière M, Ko G, Dwight M, Panopalis P, Proulx J, et al: Canadian Guidelines for the diagnosis and management of fibromyalgia syndrome. 2012. http://www. canadianpainsociety.ca/pdf/Fibromyalgia_Guidelines_2012.pdf. (accessed March, 2013).

13. Heymann RE, Paiva Edos S, Helfenstein M Jr, Pollak DF, Martinez JE, Provenza JR, Paula AP, Althoff AC, Souza EJ, Neubarth F, et al: Brazilian consensus on the treatment of fibromyalgia. Rev Bras Reumatol 2010 50(1):56-66

14. Arnold LM, Clauw DJ, McCarberg BH: Improving the recognition and diagnosis of fibromyalgia. Mayo Clin Proc 2011, 86(5):457-464.

15. Perrot S: Fibromyalgia syndrome: a relevant recent construction of an ancient condition? Curr Opin Support Palliat Care 2008, 2(2):122-127.

16. White KP, Nielson WR, Harth M, Ostbye T, Speechley M: Does the label "fibromyalgia" alter health status, function, and health service utilization? A prospective, within-group comparison in a community cohort of adults with chronic widespread pain. Arthritis Rheum 2002, 47(3):260-265. 
17. Choy E, Perrot S, Leon T, Kaplan J, Petersel D, Ginovker A, Kramer E: A patient survey of the impact of fibromyalgia and the journey to diagnosis. BMC Health Serv Res 2010, 10:102

18. Martinez JE, Ferraz MB, Sato El, Atra E: Fibromyalgia versus rheumatoid arthritis: a longitudinal comparison of the quality of life. J Rheumatol 1995, 22(2):270-274.

19. Martinez JE, Barauna Filho IS, Kubokawa K, Pedreira IS, Machado LA, Cevasco G: Evaluation of the quality of life in Brazilian women with fibromyalgia, through the medical outcome survey 36 item short-form study. Disabil Rehabil 2001, 23(2):64-68.

20. Pagano T, Matsutani LA, Ferreira EA, Marques AP, Pereira CA: Assessment of anxiety and quality of life in fibromyalgia patients. Sao Paulo Med J 2004, 122(6):252-258

21. Winkelmann A, Perrot S, Schaefer C, Ryan K, Chandran A, Sadosky A, Zlateva $\mathrm{G}$ : Impact of fibromyalgia severity on health economic costs: results from a European cross-sectional study. Appl Health Econ Health Policy 2011, 9(2):125-136.

22. Arnold LM, Crofford LJ, Mease PJ, Burgess SM, Palmer SC, Abetz L, Martin SA: Patient perspectives on the impact of fibromyalgia. Patient EduC Couns 2008, 73(1):114-120.

23. Schaefer C, Chandran A, Hufstader M, Baik R, McNett M, Goldenberg D, Gerwin R, Zlateva G: The comparative burden of mild, moderate and severe Fibromyalgia: results from a cross-sectional survey in the United States. Health Qual Life Outcomes 2011, 9:71.

24. Silverman S, Dukes EM, Johnston SS, Brandenburg NA, Sadosky A, Huse DM: The economic burden of fibromyalgia: comparative analysis with rheumatoid arthritis. Curr Med Res Opin 2009, 25(4):829-840.

25. Sicras-Mainar A, Rejas J, Navarro R, Blanca M, Morcillo A, Larios R, Velasco S, Villarroya C: Treating patients with fibromyalgia in primary care settings under routine medical practice: a claim database cost and burden of illness study. Arthritis Res Ther 2009, 11(2):R54.

26. Lachaine J, Beauchemin C, Landry PA: Clinical and economic characteristics of patients with fibromyalgia syndrome. Clin J Pain 2010, 26(4):284-290

27. Caballero-Uribe CV: The fibromyalgia problem. A Latin American point of view. Rheumatology (Oxford) 2004, 43(10):1311-1313.

28. McDonald M, DiBonaventura M, Ullman S: Musculoskeletal pain in the workforce: the effects of back, arthritis, and fibromyalgia pain on quality of life and work productivity. J Occup Environ Med 2011, 53(7):765-770.

29. Santos AM, Burti JS, Lopes JB, Scazufca M, Marques AP, Pereira RM: Prevalence of fibromyalgia and chronic widespread pain in communitydwelling elderly subjects living in São Paulo, Brazil. Maturitas 2010, 67(3):251-255

30. Fibromyalgia: facts and statistics; 2013 [http://nationalpainfoundation.org/ articles/849/facts-and-statistics].

31. De Ruddere L, Goubert L, Vervoort T, Prkachin KM, Crombez G: We discount the pain of others when pain has no medical explanation. J Pain 2012 13(12):1198-1205.

32. Häuser W, Eich W, Herrmann M, Nutzinger DO, Schiltenwolf M, Henningsen P: Fibromyalgia syndrome: classification, diagnosis, and treatment. Dtsch Arztebl Int 2009, 106(23):383-391.

33. Guymer EK, Maruff P, Littlejohn GO: Clinical characteristics of 150 consecutive fibromyalgia patients attending an Australian public hospital clinic. Int J Rheum Dis 2012, 15(4):348-357.

34. Martinez JE, Cruz CG, Aranda C, Boulos FC, Lagoa LA: Disease perceptions of Brazilian fibromyalgia patients: do they resemble perceptions from other countries? Int J Rehabil Res 2003, 26(3):223-227.

35. Zatzick DF, Dimsdale JE: Cultural variations in response to painful stimuli. Psychosom Med 1990, 52(5):544-557.

36. Riley JL 3rd, Wade JB, Myers CD, Sheffield D, Papas RK, Price DD: Racial/ethnic differences in the experience of chronic pain. Pain 2002, 100(3):291-298.

37. Rahim-Williams B, Riley JL 3rd, Williams AK, Fillingim RB: A quantitative review of ethnic group differences in experimental pain response: do biology, psychology, and culture matter? Pain Med 2012, 13(4):522-540.

38. Vlaar AP, ten Klooster PM, Taal E, Gheith RE, El-Garf AK, Rasker JJ, van de Laar MA: A cross-cultural study of pain intensity in Egyptian and Dutch women with rheumatoid arthritis. J Pain 2007, 8(9):730-736.

39. Zborowski M: Cultural components in responses to pain. J Soc Issues 1952, 8(4):16-30. (4):16-30

40. Zola IK: Culture and Symptoms-An Analysis of Patients' Presenting Complaints. Am Sociol Rev 1966, 31(5):615-630.
41. Lipton JA, Marbach JJ: Ethnicity and the pain experience. Soc Sci Med 1984, 19(12):1279-1298.

42. Todd KH, Lee T, Hoffman JR: The effect of ethnicity on physician estimates of pain severity in patients with isolated extremity trauma. JAMA 1994, 271(12):925-928.

43. Pereira AM, Valim V, Zandonade E, Ciconelli RM: Prevalence of musculoskeletal manifestations in the adult Brazilian population: a study using COPCORD questionnaires. Clin Exp Rheumatol 2009, 27(1):42-46.

44. Sanudo B, Galiano D, Carrasco L, de Hoyo M, McVeigh JG: Effects of a prolonged exercise program on key health outcomes in women with fibromyalgia: a randomized controlled trial. J Rehabil Med 2011, 43(6):521-526.

45. Jones KD, Liptan GL: Exercise interventions in fibromyalgia: clinical applications from the evidence. Rheum Dis Clin North Am 2009, 35(2):373-391.

46. Häuser W, Jung E, Erbsloh-Moller B, Gesmann M, Kuhn-Becker H, Petermann F, Langhorst J, Thoma R, Weiss T, Wolfe F, et al: The German fibromyalgia consumer reports - a cross-sectional survey. BMC Musculoskelet Disord 2012, 13:74.

47. Ng B, Dimsdale JE, Rollnik JD, Shapiro H: The effect of ethnicity on prescriptions for patient-controlled analgesia for post-operative pain. Pain 1996, 66(1):9-12.

48. Reisine S, Fifield J, Walsh SJ, Feinn R: Do employment and family work affect the health status of women with fibromyalgia? J Rheumatol 2003, 30(9):2045-2053.

49. Reisine S, Fifield J, Walsh S, Forrest DD: Employment and health status changes among women with fibromyalgia: a five-year study. Arthritis Rheum 2008, 59(12):1735-1741.

50. Sim J, Adams N: Therapeutic approaches to fibromyalgia syndrome in the United Kingdom: a survey of occupational therapists and physical therapists. Eur J Pain 2003, 7(2):173-180.

51. Perrot S, Choy E, Petersel D, Ginovker A, Kramer E: Survey of physician experiences and perceptions about the diagnosis and treatment of fibromyalgia. BMC Health Serv Res 2012, 12:356.

52. Buskila D, Neumann L, Sibirski D, Shvartzman P: Awareness of diagnostic and clinical features of fibromyalgia among family physicians. Fam Pract 1997, 14(3):238-241.

53. Acevedo JC, Amaya A, Casasola Ode L, Chinchilla N, De Giorgis M, Florez S, Genis MA, Gomez-Barrios JV, Hernàndez JJ, Ibarra E, et al: Guidelines for the diagnosis and management of neuropathic pain: consensus of a group of Latin American experts. J Pain Palliat Care Pharmacother 2009, 23(3):261-281

54. Arnold LM, Clauw DJ, Dunegan LJ, Turk DC: A framework for fibromyalgia management for primary care providers. Mayo Clin Proc 2012, 87(5):488-496.

55. Ramos-Remus C, Salcedo-Rocha AL, Prieto-Parra RE, Galvan-Villegas F: How important is patient education? Baillieres Best Pract Res Clin Rheumatol 2000, 14(4):689-703.

56. de Miquel CA, Campayo JG, Florez MT, Arguelles JM, Tarrio EB, Montoya MG, Martin AP, Salio AM, Fuentes JV, Alberch EA, et al: Interdisciplinary consensus document for the treatment of fibromyalgia. Actas Esp Psiquiatr 2010, 38(2):108-120.

57. Monterdea S, Salvata I, Montulla y S, Fernández-Ballartb J: Validación de la versión española del Fibromyalgia Impact Questionnaire. Rev Esp Reumatol 2004, 31(9):507-513.

58. Lazaro C, Caseras X, Whizar-Lugo VM, Wenk R, Baldioceda F, Bernal R, Ovalle A, Torrubia R, Banos JE: Psychometric properties of a Spanish version of the McGill Pain Questionnaire in several Spanish-speaking countries. Clin J Pain 2001, 17(4):365-374.

59. Bennett R: The Fibromyalgia Impact Questionnaire (FIQ): a review of its development, current version, operating characteristics and uses. Clin Exp Rheumatol 2005, 23(5 Suppl 39):S154-S162.

60. Usui C, Hatta K, Aratani S, Yagishita N, Nishioka K, Kanazawa T, Ito K, Yamano Y, Nakamura H, Nakajima T, et al: The Japanese version of the 2010 American College of Rheumatology Preliminary Diagnostic Criteria for Fibromyalgia and the Fibromyalgia Symptom Scale: reliability and validity. Mod Rheumatol 2012, 22(1):40-44.

doi:10.1186/1471-2474-14-188

Cite this article as: Clark et al:: A patient and physician survey of fibromyalgia across Latin America and Europe. BMC Musculoskeletal Disorders 2013 14:188 